\title{
Work distribution and path integrals in general mean-field systems
}

\author{
A. Imparato $\left(^{*}\right)\left({ }^{* *}\right)$ and L. Peliti $\left(^{*}\right)$ \\ Dipartimento di Scienze Fisiche and Unità INFM, \\ Università "Federico II", Complesso Monte S. Angelo, I-80126 Napoli (Italy)
}

PACS. 05.70.Ln - Nonequilibrium and irreversible thermodynamics.

PACS. 05.40.-a - Fluctuation phenomena, random processes, noise, and Brownian motion.

\begin{abstract}
We consider a mean-field system described by a general collective variable $M$, driven out of equilibrium by the manipulation of a parameter $\mu$. Given a general dynamics compatible with its equilibrium distribution, we derive the evolution equation for the joint probability distribution function of $M$ and the work $W$ done on the system. We solve this equation by path integrals. We show that the Jarzynski equality holds identically for these dynamics, both at the path integral level and for the classical paths which dominate the expression in the thermodynamic limit. We discuss some implications of our results.
\end{abstract}

In a class of micromanipulation experiments a system initially in thermal equilibrium is subject to the external manipulation of a given parameter $\mu$, and the work $W$ done on it is evaluated $[1,2]$. This allows one to gather some interesting information on its structure, and in particular makes it possible to evaluate the free energy difference between the initial and final values of the manipulated parameter, by means of the Jarzynski equality [3]

$$
\left\langle\mathrm{e}^{-\beta W}\right\rangle=\mathrm{e}^{-\beta \Delta F} .
$$

In this expression, $\beta=1 / k_{\mathrm{B}} T$, where $T$ is the temperature, the average on the lhs is performed over the realizations of the process, and $\Delta F$ is the difference between the values of the equilibrium free energy $F$ for the final and the initial values of the manipulated parameter $\mu$.

In order to evaluate correctly the lhs of eq. (11) it is important to gather information on the probability distribution function of $W$ for a given protocol $\mu(t)$. In a previous work [4] we pointed out that a convenient way of achieving this goal is by considering the joint probability distribution function $\Phi_{\sigma}(W)$ of $W$ and the microscopic state $\sigma$ of the system. We considered a comparatively simple system, like a pulled biomolecule subject to kinetic barriers, and obtained the corresponding work distributions. It is clear, however, that one should consider in general more complicated systems, described by collective coordinates and by more complex stochastic equations of motions.

In a recent work [5] F. Ritort introduced a path integral representation of the work distribution for a very simple system, namely a collection of noninteracting spins, and showed how

$\left({ }^{*}\right)$ Associati INFN, Sezione di Napoli.

$\left.{ }^{* *}\right)$ Corresponding author. Email: imparato@na.infn.it 
its properties could be derived by drawing on thermodynamical analogies. Here we wish to generalize both approaches, by considering a system described by a general collective coordinate $M$, an equilibrium free energy $\mathcal{F}_{\mu}(M)$, depending on a parameter $\mu$ which is manipulated according to a protocol $\mu(t), 0 \leq t \leq t_{\mathrm{f}}$, and a general stochastic evolution described by a partial differential equation of the form

$$
\frac{\partial P}{\partial t}=\widehat{\mathcal{H}} P
$$

where $P(M, t)$ is the probability distribution function of the collective variable $M$, and $\widehat{\mathcal{H}}$ is a differential operator, depending on $\mu$, and compatible with an equilibrium distribution

$$
P_{\text {eq }}(M ; \mu) \propto \mathrm{e}^{-\beta \mathcal{F}_{\mu}(M)} .
$$

We should have, therefore, for a given $\mu$ and any value of $M$,

$$
\widehat{\mathcal{H}} P_{\text {eq }}(M ; \mu)=0 .
$$

During the manipulation of $\mu$, the evolution of the system is described by a stochastic trajectory $M(t)$, and the work done on the system reads

$$
W=\int_{0}^{t_{\mathrm{f}}} \mathrm{d} t \dot{\mu}(t) \frac{\partial \mathcal{F}_{\mu}(M(t))}{\partial \mu} .
$$

The evolution equation for the joint probability distribution $\Phi(M, W, t)$ of $M$ and $W$ can be derived by following the steps of ref. [4]:

$$
\frac{\partial \Phi}{\partial t}=\widehat{\mathcal{H}} \Phi-\dot{\mu} \frac{\partial \mathcal{F}_{\mu}}{\partial \mu} \frac{\partial \Phi}{\partial W} .
$$

A similar equation for the joint probability distribution of the microscopic state and the work has been derived in ref. [6]. It is actually more convenient to introduce the corresponding generating function $\Psi(M, \lambda, t)$ for the work distribution:

$$
\Psi(M, \lambda, t)=\int \mathrm{d} W \mathrm{e}^{-\lambda W} \Phi(M, W, t) .
$$

One can readily see that $\Psi$ satisfies the following evolution equation:

$$
\frac{\partial \Psi}{\partial t}=\widehat{\mathcal{H}} \Psi-\lambda \dot{\mu} \frac{\partial \mathcal{F}_{\mu}}{\partial \mu} \Psi
$$

Thus, if $\lambda=\beta$,

$$
\mathcal{P}(M, t)=\exp \left[-\beta \mathcal{F}_{\mu(t)}(M)\right] / Z_{0},
$$

(where $Z_{0}$ is the partition function of the initial state) solves this equation and satisfies its initial condition

$$
\Psi(M, \lambda=\beta, 0)=\mathcal{P}(M, 0)=\frac{\mathrm{e}^{-\beta \mathcal{F}_{\mu(0)}}}{Z_{0}} .
$$

Thus $\Psi(M, \lambda=\beta, t)=\mathcal{P}(M, t),[7]$ and by integrating this function upon $M$ one obtains the Jarzynski equality (11). We have thus shown that the solution of eq. (6) satisfies the Jarzynski equality identically. 
In order to obtain $\Phi$, one can attempt to solve directly eq. (8) by numerical means. This approach runs into numerical difficulties when the system size $N$ becomes large, because the corresponding distributions become narrower and narrower. Much insight is gained by looking at its path integral solution in the thermodynamic limit, as pointed out in ref. [5]. By going through the steps explained, e.g., in [8], one can see that, if the differential operator $\widehat{\mathcal{H}}$ is given by

$$
\widehat{\mathcal{H}} \cdot=\sum_{k=0}^{\infty} \frac{\partial^{k}}{\partial M^{k}}\left\{g_{k}(M) \cdot\right\},
$$

one has

$$
\Psi(M, \lambda, t)=\int \mathrm{d} M_{0} \int_{M(0)=M_{0}}^{M(t)=M} \mathcal{D} \gamma \mathcal{D} M \exp \left\{\int \mathrm{d} t^{\prime} \mathcal{L}\left(t^{\prime}\right)\right\} \Psi\left(M_{0}, \lambda, 0\right),
$$

where the Lagrangian $\mathcal{L}$ is given by

$$
\mathcal{L}(t)=\left.\left(\gamma \dot{M}+\mathcal{H}(\gamma, M)-\lambda \dot{\mu} \frac{\partial \mathcal{F}_{\mu}}{\partial \mu}\right)\right|_{\gamma(t), M(t), \mu(t)} .
$$

Here $\mathcal{H}(\gamma, M)$ is given in terms of the differential operator (11),

$$
\mathcal{H}(\gamma, M)=\sum_{k=0}^{\infty} \gamma^{k} g_{k}(M, \mu(t)),
$$

and the path integral is defined by

$$
\int \mathcal{D} \gamma \mathcal{D} M=\lim _{\substack{\Delta \rightarrow 0, \mathcal{N} \rightarrow \infty \\ \mathcal{N} \Delta t=t}} \prod_{k=1}^{\mathcal{N}} \frac{\mathrm{d} \gamma(k \Delta t) \mathrm{d} M(k \Delta t)}{2 \pi \mathrm{i}}
$$

It can be readily seen that this formulation encompasses that discussed in ref. [5].

We denote by $N$ the size of the system, and set $M=N m, \mathcal{F}_{\mu}(M)=N f_{\mu}(m), \mathcal{H}(\gamma, M)=$ $N H(\gamma, m)$. As $N \rightarrow \infty$, the path integral is dominated by the classical paths $\left(\gamma_{\mathrm{c}}(t), m_{\mathrm{c}}(t)\right)$, satisfying the equations of motion

$$
\begin{aligned}
& 0=\dot{m}+\frac{\partial H}{\partial \gamma} \\
& 0=-\dot{\gamma}+\frac{\partial H}{\partial m}-\lambda \dot{\mu} \frac{\partial^{2} f_{\mu}}{\partial m \partial \mu} .
\end{aligned}
$$

The condition at $t=0$ for these equations of motion must now be discussed. For all values of $\lambda, \Psi(M, \lambda, t=0)$ satisfies eq. (10). Thus the integral over the variables at $t=0$ takes the form

$$
\Psi(M, \lambda, t=0)=\frac{\mathrm{e}^{-\beta \mathcal{F}_{\mu(0)}\left(M_{0}\right)}}{Z_{0}}=\int \frac{\mathrm{d} \gamma_{0} \mathrm{~d} M_{0}^{\prime}}{2 \pi \mathrm{i}} \mathrm{e}^{\gamma_{0}\left(M_{0}-M_{0}^{\prime}\right)} \frac{\mathrm{e}^{-\beta \mathcal{F}_{\mu(0)}\left(M_{0}^{\prime}\right)}}{Z_{0}} .
$$

Evaluating this integral by the saddle point method one obtains $m_{0}^{\prime}=m_{0}$ and that $\gamma_{0}$ and $m_{0}$ satisfy

$$
Q \equiv \beta^{-1} \gamma+\frac{\partial f_{\mu}}{\partial m}=0
$$

Thus, for any given value of $m_{0}$ one can evaluate the corresponding value of $\gamma_{0}$ by imposing eq. (19) at $t=0$, and then integrate the equations of motion (16,17) for $0 \leq t \leq t_{\mathrm{f}}$. This 
will yield the values $\left(m_{\mathrm{f}}, \gamma_{\mathrm{f}}\right)$ of $m\left(t_{\mathrm{f}}\right)$ and $\gamma\left(t_{\mathrm{f}}\right)$ respectively. By evaluating the corresponding action one can thus obtain the value of $\Psi\left(N m_{\mathrm{f}}, \lambda, t_{\mathrm{f}}\right)$. The generating functional of the work distribution $\Gamma\left(\lambda, t_{\mathrm{f}}\right)$ is given by the integral of $\Psi\left(N m_{\mathrm{f}}, \lambda, t_{\mathrm{f}}\right)$ with respect to $m_{\mathrm{f}}$, see eq. (7). As pointed out in [5], this corresponds to evaluating $\Psi\left(N m_{\mathrm{f}}, \lambda, t_{\mathrm{f}}\right)$ for that particular solution of the equations of motion which satisfies $\gamma_{\mathrm{f}}=0$. Thus one must solve the equations of motion (16).17) by imposing the boundary condition (19) at $t=0$ and the other condition $\gamma(t)=0$ at $t=t_{\mathrm{f}}$. This can be done by a shooting method: one solves the equations as a function of the initial value $m_{0}$ of $m$ and chooses that value $m_{0}^{*}$ for which $\gamma\left(t_{\mathrm{f}}\right)=0$. (This had not been necessary in ref. [5], since in that case the equation for $\gamma$ did not involve $m$.) This can be done more easily if one observes that, for $\lambda=0$, the solution $\gamma(t) \equiv 0$ is the correct one. One can build on it to evaluate successively the solution for slowly increasing values of $|\lambda|$. Alternatively, one may resort to a relaxation method, which is more convenient for slower manipulation protocols. Once the generating function $\Gamma\left(\lambda, t_{\mathrm{f}}\right)$ of the work distribution is obtained, the distribution itself can be obtained by a saddle point integration, which amounts to a Legendre transformation on $\log \Gamma$ :

$$
P\left(W, t_{\mathrm{f}}\right)=\int \mathrm{d} \lambda \mathrm{e}^{\lambda W+\log \left[\Gamma\left(\lambda, t_{\mathrm{f}}\right)\right]}=\mathrm{e}^{\lambda^{*} W+\log \left[\Gamma\left(\lambda^{*}, t_{\mathrm{f}}\right)\right]},
$$

where $\lambda^{*}$ is implicitly defined by $\left.\partial_{\lambda} \log \Gamma\right|_{\lambda^{*}}=-W$. It is worth noting that we obtain in this way a probability distribution of the work per spin, $w=W / N$, which has the form $P(w) \propto \exp [N g(w)]$, where $g(w)$ is a function of $w$ alone.

We now show that the solution obtained in this way satisfies the Jarzynski equality identically. We first show that, for $\lambda=\beta$, the solution of the classical equations of motion (16, 17) satisfy an equation analogous to (19) at all times. By multiplying both sides of eq. (4) by $\mathrm{e}^{-\gamma M}$ and integrating by parts over $M$ one obtains

$$
\int \mathrm{d} M \mathcal{H}(\gamma, M) \mathrm{e}^{-\beta \mathcal{F}_{\mu}(M)-\gamma M}=0,
$$

where $\mathcal{H}(\gamma, M)$ is given by (14). Evaluating this integral by the saddle point method in the large $N$ limit, we obtain

$$
H\left(\gamma, m^{*}\right)=0,
$$

if $\gamma$ and $m^{*}$ are related by (19). By differentiating eq. (22) with respect to $\gamma$ at fixed $\mu$ we obtain

$$
\left.\frac{\partial H}{\partial \gamma}+\left.\frac{\partial H}{\partial m}\right|_{m^{*}} \frac{\partial m^{*}}{\partial \gamma}\right)_{\mu}=0
$$

Let us also take the derivative of eq. (19) with respect to $\gamma$ at fixed $\mu$, we obtain

$$
\left.-\beta^{-1}=\frac{\partial^{2} f_{\mu}}{\partial m^{2}} \frac{\partial m^{*}}{\partial \gamma}\right)_{\mu}
$$

By multiplying both sides of eq. (23) by $\partial^{2} f_{\mu} / \partial m^{2}$ and substituting eq. (24), we obtain the following relation

$$
\frac{\partial^{2} f_{\mu}}{\partial m^{2}} \frac{\partial H}{\partial \gamma}-\beta^{-1} \frac{\partial H}{\partial m}=0
$$

which holds when $\gamma$ and $m$ are related by eq. (19). We can now evaluate the time derivative of the lhs of eq. (19), when $\gamma$ and $m$ satisfy eqs. (16, 17). We have

$$
\dot{Q}=-\beta^{-1} \dot{\gamma}-\frac{\partial^{2} f_{\mu}}{\partial m^{2}} \dot{m}-\frac{\partial^{2} f_{\mu}}{\partial m \partial \mu} \dot{\mu}=-\beta^{-1}\left(\frac{\partial H}{\partial m}-\beta \frac{\partial^{2} f_{\mu}}{\partial m \partial \mu} \dot{\mu}\right)+\frac{\partial^{2} f_{\mu}}{\partial m^{2}} \frac{\partial H}{\partial \gamma}-\frac{\partial^{2} f_{\mu}}{\partial m \partial \mu} \dot{\mu} .
$$


The second and the last term cancel out. Substituting eq. (25), we see that also the first and the third therm cancel out. Thus if $\gamma$ and $m$ satisfy eqs. (16].17) at all times, and satisfy eq. (19) at a given time, they satisfy this last equation at any time.

Thus, for $\lambda=\beta$, the Lagrangian, evaluated along the classical path, is given by

$$
\mathcal{L}_{\mathrm{c}}=N\left[\gamma \dot{m}-\beta \dot{\mu} \frac{\partial f_{\mu}}{\partial \mu}\right]=N\left[-\beta \frac{\partial f_{\mu}}{\partial m} \dot{m}-\beta \frac{\partial f_{\mu}}{\partial \mu} \dot{\mu}\right]=-\beta N \frac{\mathrm{d} f_{\mu}}{\mathrm{d} t},
$$

where we have exploited eq. (19). Substituting this expression in eq. (12) one recovers eq. (9) and the Jarzynski equality.

In the following we consider a simple mean-field Ising-like system, with free energy

$$
\mathcal{F}(M)=-\frac{J}{2 N} M^{2}-h M-T S(M),
$$

where

$$
S(M)=N k_{\mathrm{B}}\left[\ln 2-\frac{1}{2} \ln \left(1-m^{2}\right)-\frac{m}{2} \ln \left(\frac{1+m}{1-m}\right)\right] .
$$

The evolution of the system is governed by a Fokker-Planck equation with differential operator

$$
\widehat{\mathcal{H}} \cdot=\frac{\partial}{\partial M}\left[\omega_{0}\left(\frac{\partial \mathcal{F}}{\partial M}\right) \cdot+\beta^{-1} \omega_{0} \frac{\partial}{\partial M} \cdot\right] \cdot
$$

We show in fig. 1 the results of our calculation for this system, with $\beta=1, J=0.5, \omega_{0} \equiv 1$, $N=50$, and where the external field $h$ is manipulated according to the protocol

$$
h(t)=h_{0}+\left(h_{1}-h_{0}\right) \frac{t}{t_{\mathrm{f}}} ; \quad 0<t<t_{\mathrm{f}} .
$$

We have set $h_{0}=-h_{1}=-1, t_{\mathrm{f}}=2$. We compare the predictions with the results of the simulation of the Langevin equation underlying eq. (30), integrated according to the Heun algorithm [9]. We see that in this case the agreement between the simulation results and the distribution obtained by our analysis is quite good: nevertheless, one can also conclude that even for such a small system the possibility of using the results of the nonequilibrium manipulation protocol to obtain information on the equilibrium free energy is rather remote. Indeed, we have plotted in fig.11 the quantity $\Omega(w)=P(w) \mathrm{e}^{-\beta N w}$, whose integral should give $\mathrm{e}^{-\beta \Delta F}$ (which is equal to 1 in our case). We can see that not a single point among the simulated 10000 falls in the range in which $\Omega(w)$ is essentially different from 0 .

When $h=0$ and $\beta J>1$, our system undergoes a symmetry-breaking phase transition. Now assume that $h=0, \beta J(0)<1, \beta J\left(t_{\mathrm{f}}\right)>1$. Since $\gamma\left(t_{\mathrm{f}}\right)=0$, the identically vanishing solution $\left(\gamma_{\mathrm{c}} \equiv 0, m_{\mathrm{c}} \equiv 0\right)$ satisfies the equations of motion (16, 17) and the initial condition. This would lead to $W \equiv 0$ in contradiction with the Jarzynski equality. However, this solution does not correspond to the extremum of the exponent in eq. (21), which is given by the symmetry-breaking solution. It is therefore necessary to perform a quasi-average, by introducing a small symmetry-breaking field $h$, evaluating the classical action, and going to the limit $h \rightarrow 0$ at the end of the calculation. We show in fig. 2 the results of this calculation. We have set $h_{0}=h_{1}=0.01$, and manipulated $J$ according to the protocol

$$
J(t)=J_{0}+\left(J_{1}-J_{0}\right) \frac{t}{t_{\mathrm{f}}}, \quad 0<t<t_{\mathrm{f}},
$$

with $J_{0}=0.5, J_{1}=1.5, t_{\mathrm{f}}=10, \beta=1$. Notice that for such a protocol the work done on the system is non-positive, since $w=-\int \mathrm{d} t \dot{J}(t) m^{2}(t)$. The figure contains the results of 10000 


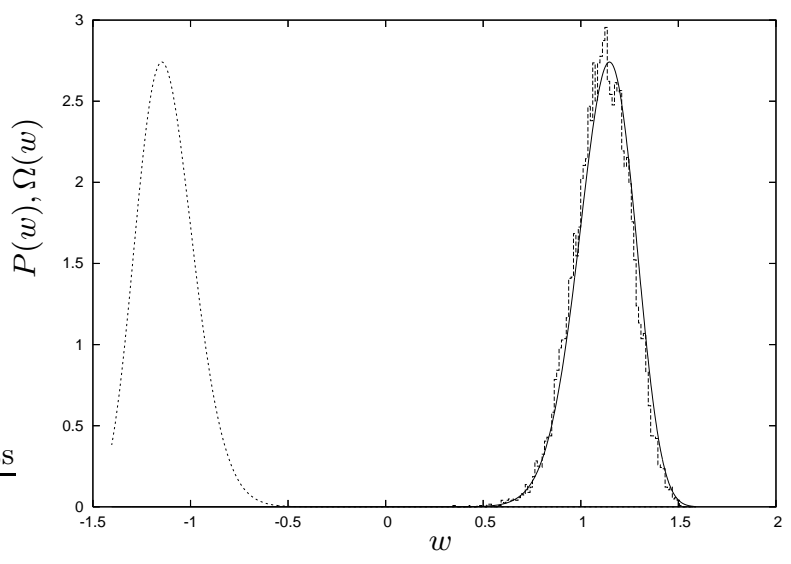

Fig. 1 - Results for the system described by the differential operator (30) with equilibrium free energy (28), manipulated according to the protocol (31). Continuous line: probability density $P(w)$ of the work "per spin" $w=W / N$, with $\mathrm{N}=50$. Dashed line: histogram of the work obtained by 10000 simulations of the process via the Heun algorithm [9]. Dotted line: $\Omega(w)=P(w) \exp (-\beta N w)$, whose integral verifies the Jarzynski equality. One can see that there are no simulation points in the range in which $\Omega(w)$ is essentially different from zero. Thus, in this case, a quantitative verification of the Jarzynski equality would be practically impossible.

simulations each with $N=1$ (continuous line), $N=10$ (dashed line), $N=100$ (dotted line), and the expectations of the theory for $N=100$ (dash-dotted line). The agreement between theory and simulations is poor for the smaller values of $N$, reasonable for $N=100$, and improves as $N$ grows (other data not shown). However, notice that for small values of $N$ the simulated values of $w$ are consistently smaller than those obtained asymptotically for large $N$, and lie closer to the thermodynamical value $w_{\text {rev }}$ denoted by a vertical line. Indeed, for small values of $h$ and large values of $N$ the mean-field system has difficulties in keeping close to the true thermodynamic minimum, whereas a smaller system can reach it by activated processes.

We have derived the differential equation (6) for the joint probability distribution of the collective coordinate $M$ and the work $W$ for a system evolving according to a general dynamics. We have shown that the solution of this equation satisfies the Jarzynski equality identically. We find that this equation can be solved by the path integral (12). This solution can be evaluated, asymptotically for large system sizes $N$, by a saddle-point integration, leading to a two-point boundary value problem for the solution of the classical equations of motions, thus generalizing the approach of ref. [5] to interacting systems. We have seen that the Jarzynski equality can be effectively exploited to obtain the free energy difference only for very small systems. We have pointed out that the method has to be handled carefully if the manipulation protocol leads in the vicinity of a phase transition. (The situation is even more delicate if the protocol crosses a discontinuous transition line.) It is possible to extend the approach to disordered mean-field systems like the Hopfield model. By allowing one to explore the work probability distribution function, the formalism can be of use in identifying the manipulation protocol most likely to yield the desired results. 


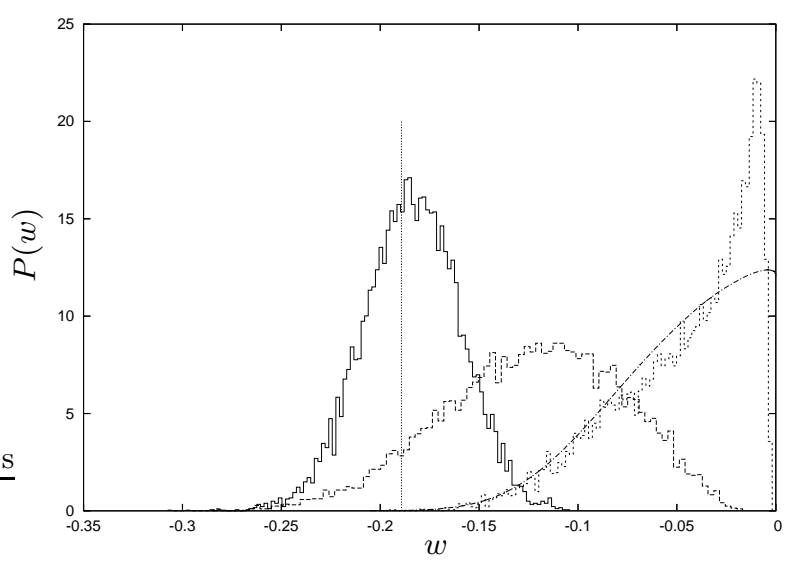

Fig. 2 - Results for the system described by the differential operator (30) with equilibrium free energy (28), manipulated according to the protocol (32). Histograms over 10000 simulations for each value of $N$. Continuous line: $N=1$. Dashed line: $N=10$. Dotted line $N=100$. Dash-dotted line: Calculated $P(w)$ for $N=100$. The calculated $P(w)$ (not shown) for smaller values of $N$ have the same general behavior as for $N=100$, but are wider. Vertical line: Thermodynamic value of the work $w_{\text {rev }}=\Delta F / N$.

We are grateful to Felix Ritort for a critical reading of the manuscript. LP is grateful to Silvio Franz for thought-provoking remarks.

\section{REFERENCES}

[1] Liphardt J., Onoa B., Smith S. B., Tinoco I. and Bustamante C., Science, 292 (2001) 733; Ritort F., Bustamante C., and Tinoco I. Jr., Proc. Natl. Acad. Sci. USA, 99 (2002) 13544; Onoa B., Dumont S., Liphardt J., Smith S. B., Tinoco I. and Bustamante C., Science, 299 (2003) 1892; Gore J., Ritort F. and C. Bustamante, Proc. Natl. Acad. Sci. USA, 100 (2003) 12564.

[2] Wang G. M., Sevick E. M., Mittag E., Searles D. J. and Evans D. J., Phys. Rev. Lett., 89 (2002) 050601; Carberry D. M., Reid J. C., Wang G. M., Sevick E. M. , Searles D. J. and Evans D. J., Phys. Rev. Lett., 92 (2004) 140601.

[3] Jarzynski C., Phys. Rev. Lett., 78 (1997) 2690; Jarzynski C., Phys. Rev. E, 56 (1997) 5018; Crooks G. E., J. Stat. Phys., 90 (1998) 1481; Crooks G. E., Phys. Rev. E, 61 (1999) 2361.

[4] Imparato A. and Peliti L., Europhys. Lett., 69 (2005) 643.

[5] Ritort F., J. Stat. Mech., (2004) P10016.

[6] Speck T. and Seifert U., Phys. Rev. E, 70 (2004) 066112.

[7] Jarzynski C., Dynamics of Dissipation, Lecture Notes in Physics, edited by Garbaczewski P. and OlKIEwicZ R., Vol. 597 (Springer-Verlag, Berlin) 2002.

[8] Feynman R. P. and Hibbs A. R., Quantum Mechanics and Path Integrals (McGraw-Hill, New York) 1965.

[9] See, e.g., Greiner A., Strittmatter W. and Honerkamp J., J. Stat. Phys., 51 (1988) 95 and Mannella R., Int. J. Mod. Phys. C, 13 (2002) 1177. 